\title{
Adult respiratory distress syndrome
}

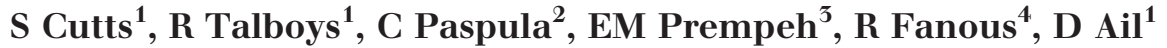 \\ ${ }^{1}$ James Paget Hospital NHS Foundation Trust, Great Yarmouth, UK \\ ${ }^{2}$ Kings Lynn Hospital, Kings Lynn, UK \\ ${ }^{3}$ Norfolk and Norwich NHS Trust, UK \\ ${ }^{4}$ London North West Healthcare Trust, UK \\ ABSTRACT
}

Adult respiratory distress syndrome (ARDS) has now been described as a sequela to such diverse conditions as burns, amniotic fluid embolism, acute pancreatitis, trauma, sepsis and damage as a result of elective surgery in general.

Patients with ARDS require immediate intubation, with the average patient now being ventilated for between 8 and 11 days. While the acute management of ARDS is conducted by the critical care team, almost any surgical patient can be affected by the condition and we believe that it is important that a broader spectrum of hospital doctors gain an understanding of the nature of the pathology and its current treatment.

\section{KEYWORDS}

Adult respiratory distress syndrome - Non-cardiogenic pulmonary oedema - 'Nam lung' - Danang lung - Shock lung - Wet lung - White

Accepted 3 July 2016

\section{CORRESPONDENCE TO}

Steven Cutts, E: stevenfrcs@hotmail.com

\section{Introduction}

In 1967, Ashbaugh ${ }^{1}$ first described adult respiratory distress syndrome (ARDS) in The Lancet. His paper had previously been rejected by the American journals, who interpreted it as another case of left ventricular failure (LVF). The condition would later feature prominently in the Vietnam war, where helicopter evacuation of the wounded enabled patients to reach an intensive care-like environment within less than an hour of injury. Patients with no apparent chest wound developed a persistent hypoxia that failed to respond to an oxygen mask and a chest $\mathrm{x}$-ray appearance reminiscent of LVF in the elderly. This observation would later provide ARDS with one of its many synonyms: non-cardiogenic pulmonary oedema.

To this day, ARDS remains a life-threatening complication that is treated by intubation and positive pressure ventilation. The apparent reduction in lung compliance is reminiscent of the respiratory distress syndrome seen in preterm neonates.

ARDS has now been described as a sequela to such diverse conditions as burns, amniotic fluid embolism, acute pancreatitis, trauma and sepsis. In modern civilian practice, it is usually associated with elective surgery.

\section{The etiology of ARDS}

The acute phase of ARDS is associated with diffuse alveolar damage and lung capillary endothelial injury. White cells adhere to the pulmonary capillaries and bore holes through the basement membrane. Large plasma proteins (such as albumin) are then able to escape into the interstitial fluid, disturbing Starling equilibrium and provoking a diffuse pulmonary oedema that extends into the alveoli, reducing both gas exchange and lung compliance. The cells in the alveoli epithelium consist of two types. ${ }^{2}$ The type I cell is a complex branched cell with multiple cytoplasmic plates that are greatly attenuated and relatively devoid of organelles; these plates represent the gas exchange surface in the alveolus. Damage to these cells allows both increased entry of fluid into the alveoli and decreased clearance of fluid from the alveolar space.

Type II alveolar epithelial cells are more resistant to injury. However, type II cells manufacture surfactant and damage to these cells results in an increase in lung compliance. These cells also act as progenitor cells for both cell lineages. Neutrophils and T-lymphocytes migrate into the inflamed lung tissue. The typical histological presentation includes diffuse alveolar damage and hyaline membrane formation in alveolar walls. The hypoxia that follows is soon exacerbated by marked intrapulmonary shunting.

\section{Diagnosis}

On plain chest $\mathrm{x}$-Ray, ARDS appears to have a far more diffuse opacity than that associated with congestive heart failure. $^{3}$ This appearance provided the condition with yet another name: white lung. However, computed tomography 
(CT) demonstrates a pulmonary picture that is anything but homogeneous. ${ }^{4}$ One of the great challenges in this environment is differentiating between LVF and ARDS in those patients where both differentials are credible.

Traditionally, thermodilution techniques were used to diagnose ARDS, using a right atrial cardiac catheter. Thermodilution involves injecting a bolus of cold saline into the pulmonary artery and looking for temperature changes in the emergent blood flow.

For many intensivists, ultrasound has become the investigation of choice in ARDS. It can be performed at the bedside without moving the patient. ${ }^{5}$ Bass et al reported that ARDS can be diagnosed using ultrasound and pulse oximetry in a low-resource environment. ${ }^{6}$

\section{Berlin definition of ARDS}

The numbers of patients suffering from ARDS is relatively small and many published studies rely on the concept of multicentre trials and or meta-analysis to achieve significant scale. Given that the definition of ARDS has changed repeatedly, it can be difficult to find equivalent studies. In 1994, (some 30 years after the Ashbaugh's original description) ARDS was given a new definition by the American European Consensus Conference (AECC). This system subdivided patients into formal ARDS and a second group who were said to suffer from acute lung injury (ALI). This system had several limitations.

In 2011, a joint team of European and American doctors attempted to redefine ARDS in what became known as the Berlin Definition (Table 1). The Berlin Definition divides patients into three stages of mild, moderate, and severe based on the degree of hypoxia in the patient. ${ }^{7}$ Each of these stages is associated with increased mortality. There is also a progressive increase in median duration of mechanical ventilation in survivors (compared with the previous AECC definition, the final Berlin Definition was deemed to be a better predictor of mortality).

\section{Management of ARDS}

ARDS requires urgent intubation and ventilation, careful assessment and treatment of the underlying causes and continual reassessment for potential complications. At present, no single treatment has been shown to modify the underlying pathological process of ARDS. However, by combining several supportive strategies, it is possible to improve the outcome.

\section{Ventilation}

The average patient with ARDS spends between 8 and 11 days on a mechanical ventilator. The objective is to maintain blood oxygen levels while avoiding oxygen toxicity and the complications of mechanical ventilation. Increasing the percentage of oxygen in inspired air is standard, although very high oxygen levels are toxic. Positive end-expiratory pressure (PEEP) is used in ARDS to 'recruit' atelectatic alveoli. Some of these alveoli can be recruited with minimal PEEP, while others can only be recruited with high levels of PEEP. High PEEP increases mean airway pressure and alveolar

$\begin{array}{lll}\text { Table } 1 & \text { The Berlin Definition } \\ \text { Grade } & \begin{array}{l}\mathrm{PaO}_{2} / \mathrm{FIO}_{2} \\ (\mathbf{m m H g})\end{array} & \begin{array}{l}\text { Ventilation } \\ \text { (mean days) }\end{array} \\ \text { Mild } & \leq 201-300 & 5 \\ \text { Moderate } & \leq 101-200 & 7 \\ \text { Severe } & \leq 100 & 9\end{array}$

$\mathrm{FIO}_{2}=$ fraction of inspired oxygen; $\mathrm{PaO}_{2}=$ partial pressure of arterial oxygen; a decreased $\mathrm{PaO}_{2} / \mathrm{FiO}_{2}$ ratio indicates reduced arterial oxygenation from the available inhaled gas

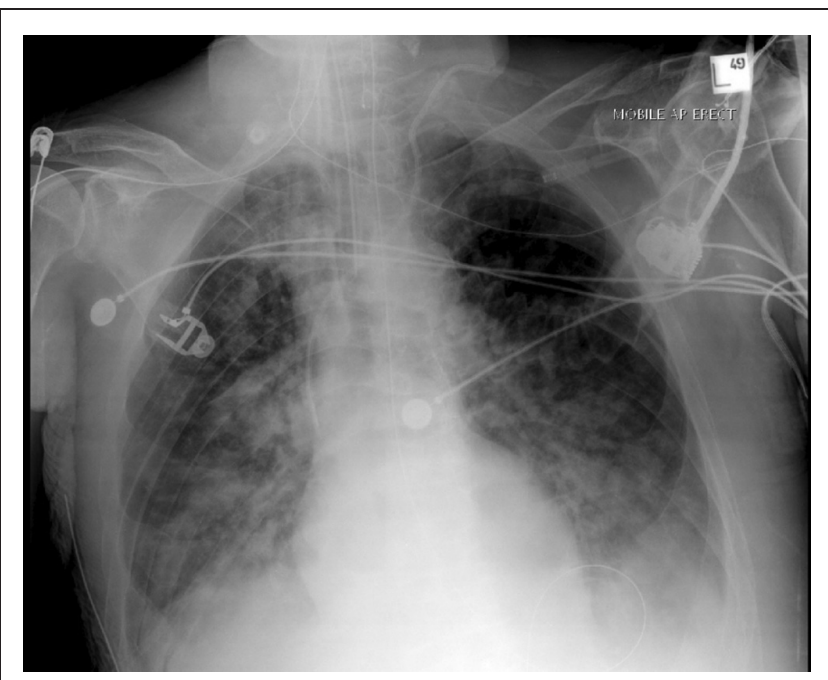

Figure 1 Plain chest $x$-ray showing adult respiratory distress syndrome

pressure, which can damage normal alveoli by overdistension, resulting in diffuse alveolar damage. It has also being suggested that reduced tidal volumes dampen or reduce the amount of inflammatory mediators released during ventilation. A mild respiratory acidosis has also being tolerated by some workers (the permissive hypercapnia), although the optimum level of acidosis permitted remains controversial.

Lung protective strategies use smaller tidal volumes to avoid barotrauma. In an ARDS network study, patients with ARDS were randomised to mechanical ventilation either at a standard or reduced tidal volume. The study was stopped early, after a mid-point analysis of 861 patients demonstrated a significantly lower mortality rate in the patients who received the lower tidal volume (31\% versus $39.8 \%$ ).

The ARDSnet trial, performed in the 1990s, suggested that mortality could be reduced by as much as $22 \%$ using low volume ventilation. This strategy also reduced the number of days spent on ventilation and associated end organ failure. However, later meta-analysis of similar studies ${ }^{8}$ failed to deliver the same result and suggested that mortality rates might even increase with low tidal volumes. 


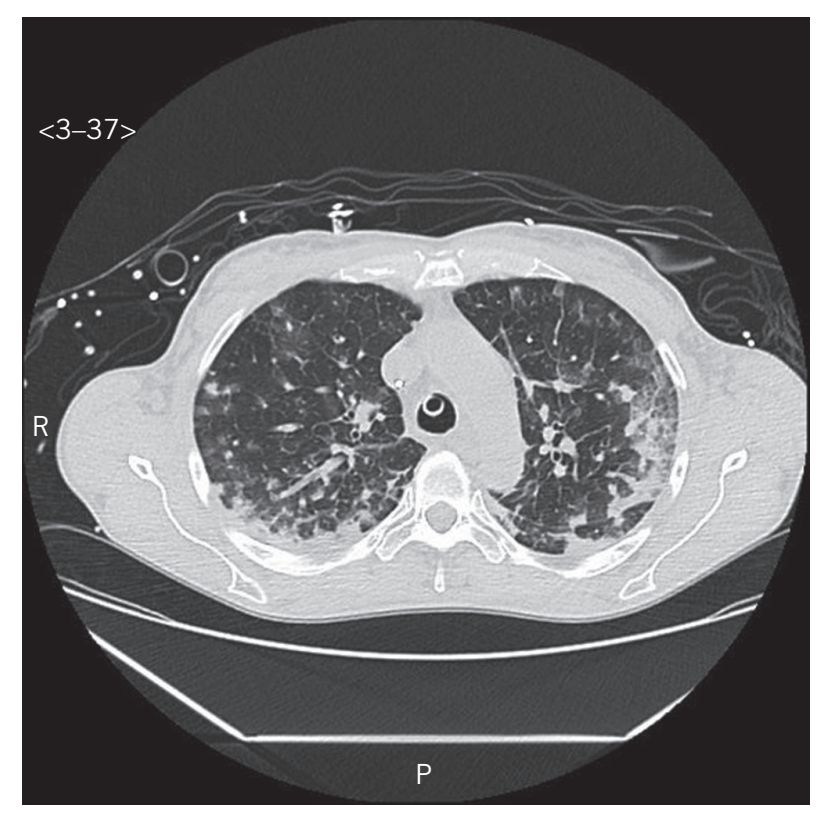

Figure 2 Computed tomography showing adult respiratory distress syndrome

Much research has been directed at jet insufflation, which attempts to move air using small successive bursts. Oxygen then diffuses down the smaller airways and into those alveoli that are still functioning. Such an approach might maintain blood oxygenation while avoiding barotrauma. In particular, jet insufflation has been reported to be successful in the treatment of children with ARDS. ${ }^{9}$

\section{Patient positioning}

Some units advocate nursing patients with ARDS in the prone position. The rationale for this is that distribution of lung infiltrates in acute respiratory distress syndrome is non-uniform. Repositioning into the prone position might improve oxygenation by relieving atelectasis and improving perfusion. A recent review on the prone position (supports this intervention. ${ }^{10}$ Pelosi performed serial CT scans of patients in intensive care units (ITU), in both the prone and supine positions. This study showed that prone ventilation redistributed the tidal volume to dorsal lung units close to the diaphragm, successfully recruiting atelectatic lung units in this region. ${ }^{11}$

Prone ventilation has also been shown to achieve an improved arterial oxygenation. Guérin et al, writing in the New England Journal of Medicine, reported a large multicentre study in France involving over 400 patients. In this study, early application of prolonged prone-positioning sessions significantly decreased 28-day and 90-day mortality. While the results of earlier trials into patient positioning produced equivocal results, the relatively large scale of the French study would appear to give it credibility. Indeed, ARDS is managed in the prone position in our own unit. ${ }^{12}$

\section{Fluid management}

Modern trauma management attempts to resuscitate the patient in the immediate aftermath of injury. Fluid management therefore attempts to address circulatory shock and the associated risk of end-organ injury. Aggressive fluid management also represents an attempt to limit the evolving inflammatory cascade. However, several studies have indicated that reducing fluid replacement and even administering diuretics to create a negative fluid balance seems to be advantageous in the first few days when treating ARDS.

An ARDS Clinical Trials Network study of a fluid-conservative strategy versus a fluid-liberal strategy in the management of patients with ARDS found no statistically significant difference in 60-day mortality between the two groups 72 hours after presentation with ARDS. However, patients treated with the fluid-conservative strategy did have improved oxygenation and lung injury scores and an increase in ventilator-free days, with no increased risk of non-pulmonary organ failures.

\section{Enhancing fluid clearance}

Resolution requires fluid clearance across the alveolar surface, and fluid clearance is critically dependent on the function of the sodium pump in the epithelial cells. Several pharmacological agents have been used to increase the rate of fluid clearance. Aerosolised beta two agonists have been used during ventilation to stimulate the sodium pump and to accelerate fluid clearance. ${ }^{13}$ Aerosolised prostacyclin has also been used in the treatment of ARDS. However, although prostacyclin has been shown to bring about pulmonary vasodilation, it has no impact on blood oxygenation or mortality. Researches have also experimented with prostaglandin E1, which is a known vasodilator. Prostaglandin E1 also downgrades neutrophil activation and early animal studies suggested that it might be effective in the treatment of ARDS. ${ }^{14}$

\section{Nitric oxide}

Inhaled nitric oxide dilates pulmonary arteries and transiently improves blood oxygenation. Rapid scavenging by haemoglobin removes nitric oxide from the circulation and prevents any impact on the systemic circulation..$^{15}$ However, nitric oxide does not reduce mortality and there is some evidence that it may be dangerous. Ketonazole has also being used in an attempt to encourage alveolar clearance. Initial reports were promising but late studies showed no improvement. ${ }^{16,17}$

\section{Corticosteroids}

In his seminal abstract, Ashbaugh ${ }^{1}$ suggested that ARDS might respond to corticosteroids. Thus far, there is no evidence to support this view.

\section{Surfactant}

Lack of surfactant contributes to atelectasis, shunts, poor gas exchange and increased rates of ventilator associated pneumonia. Although artificial surfactant is of benefit in preterm neonates, results from its use in adults have been disappointing. ${ }^{18}$ In the specific case of direct lung injury, 
analysis of the use of recombinant protein C-based surfactant in patients with ARDS suggested a trend towards a survival benefit. This effect may be related to an improvement in gas exchange.

\section{Sepsis}

Sepsis is the medical condition that is most commonly associated with ARDS. Aggressive treatment with both antibiotics and activated protein $\mathrm{C}$ is considered appropriate. ${ }^{19}$

\section{Towards a disease modifying strategy}

Since ARDS was identified, the bulk of the published research has concentrated on the management of patients with the established condition. In more recent years, there has been an attempt to better understand the underlying inflammatory changes and to introduce treatment modalities that might arrest the underlying pathological process and accelerate recovery.

Inhaled insulin has been used to suppress alveoli inflammation. Many patients in ITU are managed in a hyperglycaemic state. Inhaled insulin helps to reduce plasma hyperglycaemia, which is normally done using intravenous insulin. $^{20}$

Gene therapy and stem cell therapy have both been investigated for the treatment of ARDS. ${ }^{21}$ Early animal models suggest that gene therapy may represent a disease-modifying strategy. ${ }^{22}$

\section{Extracorporeal oxygenation techniques}

In recent years, carefully selected patients have been managed with extracorporeal oxygenation. This is expensive and the facility would not be available in all ITU units. ${ }^{23}$

Partial liquid ventilation is an exotic treatment where perfluorocarbon-filled lungs are ventilated with conventional gas mechanical ventilation. The fluid increases gas exchange by recruiting atelectatic regions of the lung, redistributing pulmonary blood flow and reducing total lung water. However, while partial liquid ventilation may be conceptually attractive, a recent Cochrane review of the field failed to find evidence of its usefulness. ${ }^{24}$

\section{Conclusions}

The average UK ITU would encounter 10-15 cases of ARDS per year. This relative paucity of numbers creates problems for researchers in this field, with most single-unit studies struggling to achieve statistically significant numbers.

While ventilation is obligatory in ARDS, the optimal level of PEEP has yet to be determined. Mechanical ventilation with smaller tidal volumes $(6 \mathrm{ml} / \mathrm{kg}$ predicted body weight) is associated with improved survival.

Prone positioning and low-dose inhaled nitric oxide both improve oxygenation and probably improve survival. Exogenous surfactant may improve oxygenation but, again, the drug has no significant effect on survival. A number of drug treatments have been tried in ARDS but, so far, none of them has been shown to improve survival. Survival in ARDS has improved significantly since the early 1990s, largely due to the advent of lung protective strategies.

The sequence of metabolic and immunological events that provokes immunocompetent cells to attack the pulmonary capillary epithelium remains unexplained. The 'holy grail' of ARDS research remains a disease modifying agent, and early animal studies suggest that there may be a future in gene therapy. No such agent is yet available to clinicians.

\section{References}

1. Ashbaugh DG, Bigelow DB, Petty TL et al. Acute respiratory distress in adults. Lancet 1967; 2(7511): 319-323.

2. Ward HE, Nicholas TE. Alveolar type I and type II cells. Aust N Z J Med 1984; 14(5 Suppl 3): 731-734.

3. Reed JC. Chest Radiology: Plain Film Patterns and Differential Diagnoses. 6th edn. Philadelphia, PA: Elsevier Mosby; 2011.

4. Caironi $P$, Carlesso E, Gattinoni L. Radiological imaging in acute lung injury and acute respiratory distress syndrome. Semin Respir Crit Care Med 2006; 27(4): 404-415.

5. Lichtenstein DA. Lung ultrasound in the critically ill. Ann Intensive Care 2014; 4(1): 1

6. Bass CM, Sajed DR, Adedipe AA et al. Pulmonary ultrasound and pulse oximetry versus chest radiography and arterial blood gas analysis for the diagnosis of acute respiratory distress syndrome: a pilot study. Crit Care 2015; 19: 282.

7. ARDS Definition Task Force, Ranieri VM, Rubenfeld GD, Thompson BT, Ferguson ND, Caldwell E, Fan E, Camporota L, Slutsky AS. Acute respiratory distress syndrome: the Berlin Definition. JAMA 2012; 307(23): 2,526-2,533.

8. Eichacker PQ, Gerstenberger EP, Banks SM et al. Meta-analysis of acute lung injury and acute respiratory distress syndrome trials testing low tidal volumes. Am J Respir Crit Care Med 2002; 166(11): 1,510-1,514.

9. Smith DW, Frankel LR, Derish MT et al. High-frequency jet ventilation in children with the adult respiratory distress syndrome complicated by pulmonary barotrauma. Pediatr Pulmonol 1993; 15(5): 279-286.

10. Kallet RH. A comprehensive review of prone position in ARDS. Respir Care 2015; 60(11): 1,660-1,687.

11. Pelosi $\mathrm{P}$, Tubiolo D, Mascheroni $\mathrm{D}$, et al. Effects of the prone position on respiratory mechanics and gas exchange during acute lung injury. Am J Respir Crit Care Med 1998; 157: 387-393.

12. Guérin $C$, Reignier J, Richard JC, et al. Prone positioning in severe acute respiratory distress syndrome. N Engl J Med 2013; 368(23): 21592168.

13. Afshari A, Brok J, Møller AM et al. Aerosolized prostacyclin for acute lung injury (ALI) and acute respiratory distress syndrome (ARDS). Cochrane Database Syst Rev 2010; 4(8): CD007733.

14. Abraham E, Baughman R, Fletcher $\mathrm{E}$ et al. Liposomal prostaglandin $\mathrm{E}_{1}$ (TLC C-53) in acute respiratory distress syndrome: a controlled, randomized, doubleblind, multicenter clinical trial; TLC C-53 ARDS Study Group. Crit Care Med 1999; 27: 1,478-1,485.

15. Afshari A, Brok J, Møller AM et al. Inhaled nitric oxide for acute respiratory distress syndrome (ARDS) and acute lung injury in children and adults. Cochrane Database Syst Rev 2010; (7): CD002787.

16. Yu M, Tomasa G. A double-blind, prospective, randomized trial of ketoconazole, a thromboxane synthetase inhibitor, in the prophylaxis of the adult respiratory distress syndrome. Crit Care Med 1993; 21: 1,635-1,642.

17. The ARDS Network. Ketoconazole for early treatment of acute lung injury and acute respiratory distress syndrome: a randomized controlled trial. JAMA 2000; 283: 1,995-2,002.

18. Baudouin SV. Surfactant medication for acute respiratory distress syndrome. Thorax 1997; 52(Suppl 3): S9-S15.

19. Chastre J, Trouillet JL, Vuagnat A et al. Nosocomial pneumonia in patients with acute respiratory distress syndrome. Am J Respir Crit Care Med 1998; 157 (4Pt1): 1,165-1,172.

20. Shapiro H, Kagan I, Shalita-Chesner M et al. Inhaled aerosolized insulin: a 'topical' anti-inflammatory treatment for acute lung injury and respiratory distress syndrome? Inflammation 2010; 33(5): 315-319.

21. Lin X, Dean DA. Gene therapy for ALI/ARDS. Crit Care Clin 2011; 27(3): 705-718.

22. Emr BM, Roy $\mathrm{S}$, Kollisch-Singule $\mathrm{M}$ et al. Electroporation-mediated gene delivery of $\mathrm{Na}^{+}, \mathrm{K}^{+}$-ATPase, and $\mathrm{ENaC}$ subunits to the lung attenuates acute 
respiratory distress syndrome in a two-hit porcine model. Shock 2015; 43(1): 16-23.

23. Belohlavek J, Rohn V, Jansa P et al. Veno-arterial ECMO in severe acute right ventricular failure with pulmonary obstructive hemodynamic pattern. J Invasive Cardiol 2010; 22(8): 365-369.
24. Galvin IM, Steel A, Pinto R et al. Partial liquid ventilation for preventing death and morbidity in adults with acute lung injury and acute respiratory distress syndrome. Cochrane Database Syst Rev 2013; (7): CD003707. 